\title{
Castles in southern Italy, diagnostic plan for knowledge and the enhancement
}

\author{
Caterina Gattuso ${ }^{\text {a }}$, Philomène Gattuso, Elena Bencardino, Valentina Caramazza
}

Dep. of Biology, Ecology and Earth Sciences, Univ. of Calabria, Rende (Cosenza), Italy, ${ }^{a}$ caterina.gattuso@unical.it

\begin{abstract}
In the south of Calabria in Italy, along the Tyrrhenian coast, lie fortifications of great historical and architectural importance which all enjoy strategic positions owing to their landscapes and wide view of the sea. Over time, such a peculiarity, which was originally the result of the need to defend the territory from foreign raids from the sea, has become a feature favouring tourist attractiveness. Worth mentioning are Sant'Aniceto fortress, the Castle of Fiumefreddo, the Castle of Cleto, and the Castle of Belvedere, which enshrine historical legacies of the Calabrian fortification system.

The study examines such architectural examples focusing not only on their historical and architectural dimension, but also on the types of construction, on the materials and on the current state of conservation of the castles concerned. Therefore, a particular diagnostic plan was developed for each building, which started from the environmental contextualization, reconstructed the anamnesis and, finally, examined constituent materials and their level of deterioration. The results of a laboratory analysis, which was carried out on micro-samples of material taken noninvasively, are shown with reference to a few representative elements.
\end{abstract}

Keywords: Castle, Calabria, knowledge.

\section{Introduzione}

Per poter conservare e valorizzare un'opera di interesse storico-architettonico è indispensabile acquisirne la conoscenza sviluppando un dettagliato piano diagnostico che consenta di redigere il quadro della situazione (Gattuso, 2001; Gattuso, 2011). La metodica utilizzata per sviluppare il piano diagnostico è stata applicata considerando un contesto territoriale sul quale insistono alcuni castelli calabresi con l'obiettivo finale di fornire gli elementi necessari per poter sviluppare azioni di valorizzazione. L'analisi degli esempi in studio ha permesso di evidenziare delle peculiarità accomunanti che rende possibile lo sviluppo della medesima metodica di studio, pertanto si è scelto di applicarla, in modo rappresentativo ad uno di essi ed in particolare al castello di Belvedere Marittimo.

\section{I castelli, il loro contesto territoriale e il profilo storico}

I castelli esaminati sono caratterizzati dall'essere situati in un contesto dal forte impatto paesaggistico, che li rende particolarmente attraenti ai fini della definizione di un piano turistico a scala territoriale.

La scelta del luogo per la costruzione del castello, effettuata in passato per esigenze difensive, diventa oggi un aspetto rilevante per la loro valorizzazione. In particolare in questo studio si è posta l'attenzione su un gruppo di castelli situati sul versante Tirrenico della Regione Calabria (Fig. 1).

Per ciascuno di essi è stata quindi elaborata una breve scheda descrittiva nella quale sono 
riportati in sintesi le fasi principali di un piano diagnostico, quindi a partire dall'esame del contesto geografico, si giunge, passando attraverso l'analisi storica ed architettonica, alla caratterizzazione dei materiali costitutivi e del loro stato di conservazione (Gattuso, 2010; Feiffer, 1989) . Le schede, di seguito riportate, sono state redatte illustrando i castelli cominciando da quello posto più a sud per giungere a quello più a nord.

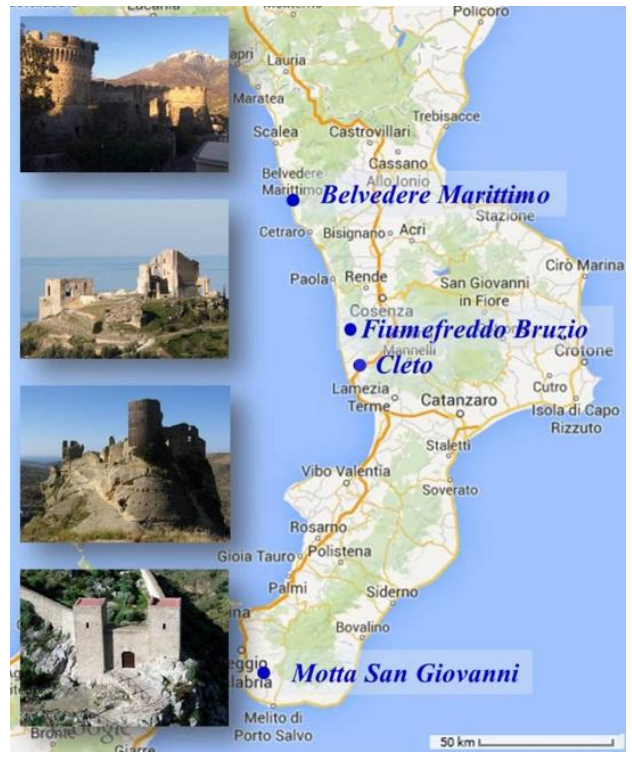

Fig. 1 - Localizzazione geografica dei castelli

Per ciascuno di essi è stata quindi elaborata una breve scheda descrittiva nella quale sono riportati in sintesi le fasi principali di un piano diagnostico, quindi a partire dall'esame del contesto geografico, si giunge, passando attraverso l'analisi storica ed architettonica, alla caratterizzazione dei materiali costitutivi e del loro stato di conservazione (Gattuso, 2010; Feiffer, 1989) . Le schede, di seguito riportate, sono state redatte illustrando i castelli cominciando da quello posto più a sud per giungere a quello più a nord.

\subsection{Castello di Sant'Aniceto a Motta San Giovanni}

Nella cittadina di Motta San Giovanni, in provincia di Reggio Calabria, sulla vetta di una montagna si trova il centro fortificato di Sant'Aniceto, costruito dai Bizantini nella prima metà del XI secolo con il fine di fronteggiare gli attacchi arabi alla costa calabra. Dopo la guida di Ruggero il normanno e di altri feudatari, durante il tormentato periodo di lotte tra Angioini ed Aragonesi, S. Aniceto passa di mano in mano fino a quando nel 1465 viene estinto.

La fortezza. Un unico accesso protetto da due torri quadrate introduce alla fortificazione dove, nel punto più alto in cui si trova una torrecisterna, un muro trasversale di sbarramento costituisce una seconda linea di difesa (Fig. 2). È in questa zona che si trovano gli edifici residenziali collegati da mura che creano una terza zona protetta.

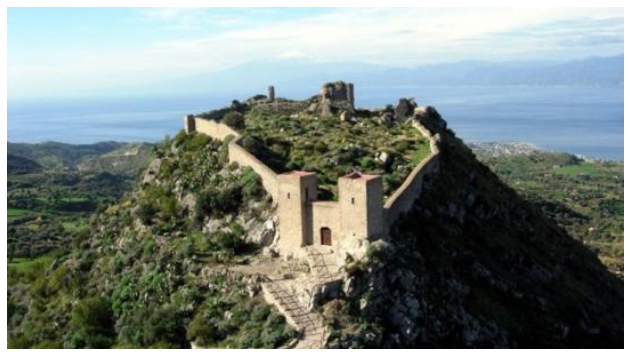

Fig. 2 - Il Castello di Sant'Aniceto

La necessità di creare un ricovero per le popolazioni e per proteggere i loro beni dagli invasori fu il motivo che li spinse a realizzare la fortificazione. Una serie di edifici di culto di modeste dimensioni, due delle quali contengono tracce ancora visibili di affreschi, la Chiesa dell'Annunziata e la Chiesa di S. Antonio, in parte crollate, segnano il percorso che porta all'ingresso della fortezza di S. Niceto. La cinta muraria, prevalentemente intatta, ha andamento irregolare ed è interrotta, a circa metà, da un muro trasversale che crea uno sbarramento e una seconda zona fortificata nel punto più alto.

Il materiale impiegato per la costruzione è costituito prevalentemente da calcarenite utilizzato anche per realizzare le mura il cui spessore medio è di circa $110 \mathrm{~cm}$. Delle due scale in pietra, l'una collocata presso l'ingresso principale (a sinistra) e l'altra nei pressi della torre del palazzo settentrionale (in basso), 
rimangono solo poche tracce. Esiste una terza scaletta sul fianco meridionale della cinta ma a differenza delle precedenti è ricavata nello spessore murario della cortina.

\subsection{Castello di Fiumefreddo Bruzio}

Fiumefreddo Bruzio, comune della provincia di Cosenza, sorge su una rocca in posizione panoramica sulla costa tirrenica e costituisce una delle più antiche cittadine del litorale. Secondo alcune fonti l'antica colonia Temesa sarebbe sorta sul litorale tra Fiumefreddo Bruzio e San Lucido. La colonia famosissima per il commercio fu distrutta nella II guerra punica da Annibale e ricostruita nel 194 a. C. dai romani, il cui dominio è attestato da molteplici tracce archeologiche. Distrutta nuovamente a causa delle invasioni Saracene i cittadini superstiti fondarono la nuova colonia sulla collina. L'antico centro che si distende su una rupe a strapiombo sul mare circondato da monti ricchi di vegetazione conserva ancora parte della cinta muraria che le rovine del castello e la Chiesa Matrice chiudono all'estremità.

$\mathrm{La}$ fortezza. Indicato nelle vecchie scritture come Castelfreddo, fu fatto edificare da Roberto il Guiscardo intorno al 1060 insieme alla cinta muraria. Un recinto di pietre lo divideva dal centro abitato mentre un ponte levatoio permetteva l'accesso da nord. L'architettura esterna seicentesca è ancora oggi in buona parte conservata (Fig. 3). Fu ridotto allo stato attuale nel 1807 quando le truppe napoleoniche lo bombardarono per conquistare il feudo. Nel corso dei secoli, il maniero venne più volte rimaneggiato e fu continuamente trasformato, in particolar modo dall'Alarcon che lo trasformò in un sontuoso e affascinante castello, dotandolo di un ampio fossato e di un ponte levatoio girevole di cui non resta traccia. Delle strutture originarie del castello, costruito interamente in pietra, restano alcuni particolari architettonici tra cui le finestre in tufo con la trabeazione di tipo classico e la costruzione su due livelli, il cui piano superiore era costituito da stanze e saloni, arricchiti da loggiati andati distrutti, alcuni rivolti verso i monti ed altri verso il mare a picco sulla rupe. Nelle sale si conservano ancora i numerosi camini in pietra lavorata ed in alcune il pavimento. I sotterranei, unica parte del castello attualmente recuperabile, erano adibiti a carcere e presentavano labirinti di cunicoli e di gallerie impenetrabili.

Le torri circolari, che nel 1500 sostituirono quelle quadrate di manifattura sveva, erano caratterizzate da merlature di tipo islamico. $\mathrm{La}$ nuova casata degli Alarcon-Mendoza governò su Fiumefreddo Bruzio fino al 1799 e oggi la proprietà del castello appartiene al Comune di Fiumefreddo Bruzio.

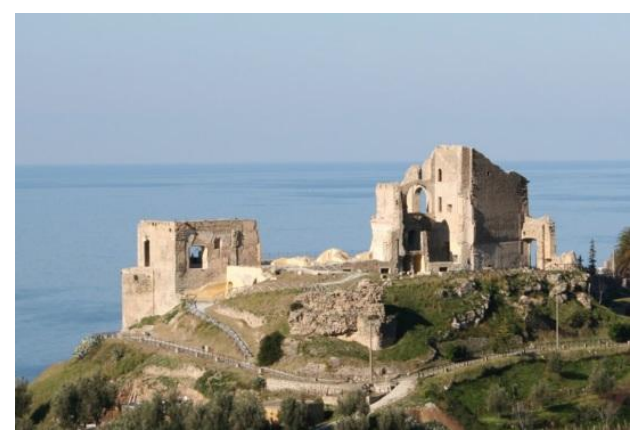

Fig. 3 - Il Castello di Fiumefreddo Bruzio

\subsection{Castello di Cleto}

Cleto è un comune della provincia di Cosenza in Calabria collocato nell'entroterra del basso tirreno cosentino tra i rilievi della catena paolana meridionale, nell'alta valle del fiume Torbido. L'abitato del comune di Cleto, è situato sulle pendici del monte S. Angelo, incastonato su di una rupe rocciosa ad un'altezza media di 250 metri s.l.m. ed è diviso in vari piccoli centri e frazioni. La presenza di reperti testimonia che la zona fu frequentata in epoche molto antiche; l'abitato anticamente noto con il nome di Pietramala o Petramala (Pietra Dura), assunse successivamente in epoca medievale il nome di Cleto. Il tessuto urbano si adatta perfettamente alla morfologia del territorio, che presenta un costone roccioso che s'innalza fino alla parte più alta dove è situato il castello che sovrasta il borgo arroccato difeso dagli strapiombi. Il borgo presentava un sistema difensivo di cinta murarie integrate da torri circolari e dalle porte 
d'accesso. Oggi le torri non sono del tutto identificabili perché sono state inglobate in strutture costruite successivamente (Fig. 4).

La fortezza. Situato nella parte più alta del centro storico di Pietramala, l'antico castello lungo circa $60 \mathrm{~m}$ e largo circa $50 \mathrm{~m}$, è collocato ad un'altezza di $360 \mathrm{~m}$ s.l.m. Il manufatto architettonico, presenta una forma trapezoidale, con due torri cilindriche a difesa dell'accesso e un bastione angolare scarpato.

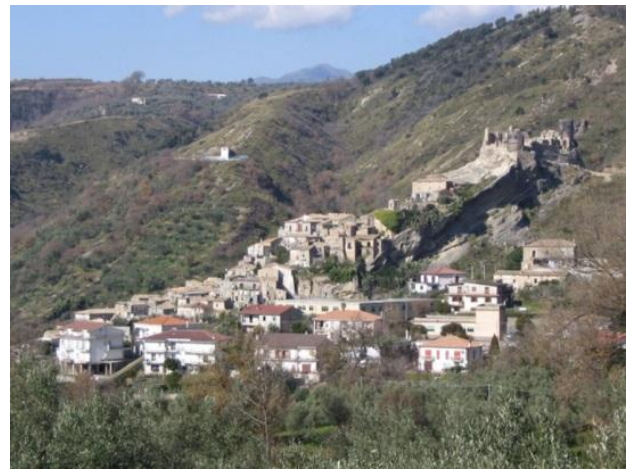

Fig. 4 - Il borgo medievale di Cleto

La planimetria è determinata dall'orografia del luogo e dalle varie ricostruzioni architettoniche succedutesi nelle varie epoche. Il castello si presenta suddiviso in due livelli: un primo livello di accesso, caratterizzato da ambienti ricavati nella roccia e un secondo superiore dove risiedeva il feudatario, come si può dedurre dalla grandezza degli ambienti tuttora visibili (Fig. 5).

Attualmente il maniero conserva in buone condizioni diverse parti, tra cui le mura perimetrali e l'imponente ingresso collocato a sud-ovest rivolto al borgo, costituito da un bastione scarpato nel lato sinistro e un avancorpo finestrato che protegge la rampa d'accesso all'arco. L'arco realizzato in conci squadrati e lavorati in pietra locale costituisce l'ingresso più recente al castello. Il più antico, posto a sud, è caratterizzato dalla presenza di un ponte levatoio raggiungibile dalla strada ricavata nella roccia. Per proteggere tale accesso, erano state costruite le due torri cilindriche.

Nonostante gli ampliamenti e i rifacimenti subiti, sono ancora leggibili molti stili architettonici di tipo militare tipici dei fortilizi, dai prototipi medievali fino a quelli propri delle fortificazioni "mature" del Viceregno.

Durante i lavori di recupero e di restauro del castello effettuati recentemente sono state rinvenute alcune cisterne e dei silos scavati nella roccia, utilizzati come contenitori di grano e acqua. Per costruire il castello è stata usata principalmente pietra locale, nello specifico arenaria e cemento calcareo, impiegata in varie forme sia come pietrame semplicemente spaccato o sbozzato che come pietra lavorata (Aiello, 2010; Placanica, 1999).

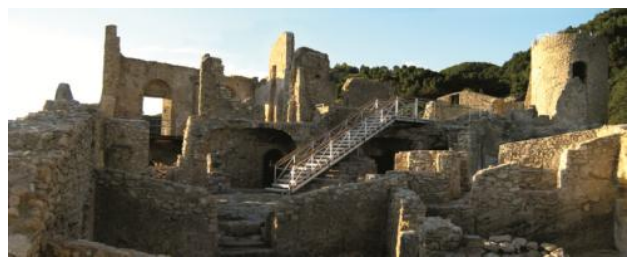

Fig. 5 - Il Castello di Cleto, interno

\section{Castello di Belvedere Marittimo}

Il centro di Belvedere Marittimo, paese Calabrese situato ai piedi del monte Montea, in un'area composta da pianure e declivi, è posto a $300 \mathrm{~m}$ sul livello del mare.

Fonti incerte fanno risalire la sua fondazione intorno all'anno mille, anche se ritrovamenti archeologici testimoniano che la zona era già popolata nei secoli precedenti. Il castello fu probabilmente ricostruito nel XI sec. da Roberto il Guiscardo, fratello di Ruggero il Normanno su strutture preesistenti. Restaurato dai vari proprietari che lo occuparono, tra questi Ruggero di Sangineto nel 1287, Ferdinando I $^{\circ}$ d'Aragona nel 1490 ed infine i Principi Sanseverino e Carafa, presenta manifatture riconducibili a diverse epoche. Le aree interne del castello, completamente ricostruite, furono adattate ad abitazione dalla famiglia Spinelli. Il castello infine, interamente restaurato grazie ai contributi dei cittadini, ha acquisito l'aspetto attuale come testimonia una lapide con due putti e un'incisione posta sul ponte levatoio (Fig. 6) (Grisolia, 1980; Rogato, 1992).

La fortezza. Il castello, realizzato con blocchi in pietra e malta di calce, presenta una pianta dalla 
forma trapezoidale ed è caratterizzato da una base a scarpata con prolungamento a parapetto sormontato da una merlatura guelfa. L'edificio poggia sul lato est su una roccia preesistente mentre sul lato sud sono poste due torri merlate. Sulla parte emergente del mastio spicca una fascia con archetti a sesto acuto sorretti da piedritti e l'altra torre coincide con la cinta muraria.

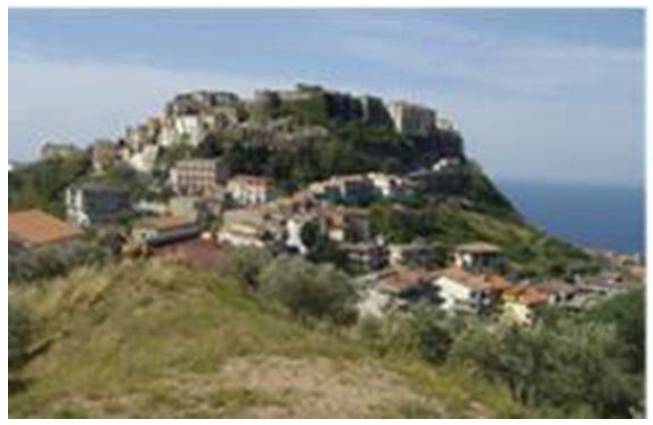

L'intera struttura, mediante una cordonatura situata a metà altezza, si suddivide in due ordini: la parte superiore che possiede una muratura a piombo sormontata da una merlatura guelfa e quella inferiore che si presenta a scarpata. Per accedere al castello veniva utilizzato un ponte levatoio a causa del fossato che lo circondava, mentre al mastio vi si poteva accedere dal cortile posto all'interno delle mura (Fig. 7).

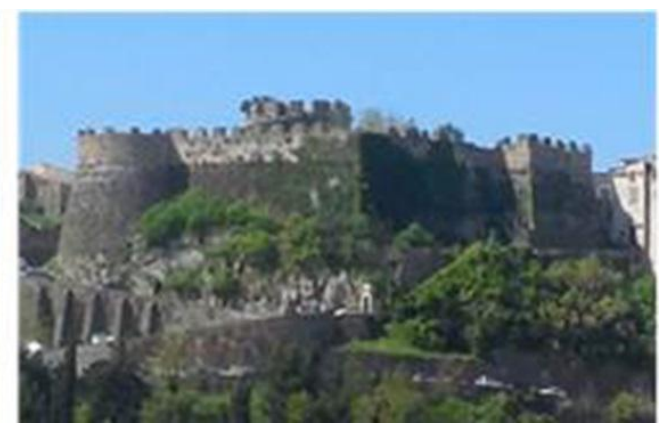

Fig. 6 - Il centro abitato e il Castello di Belvedere Marittimo

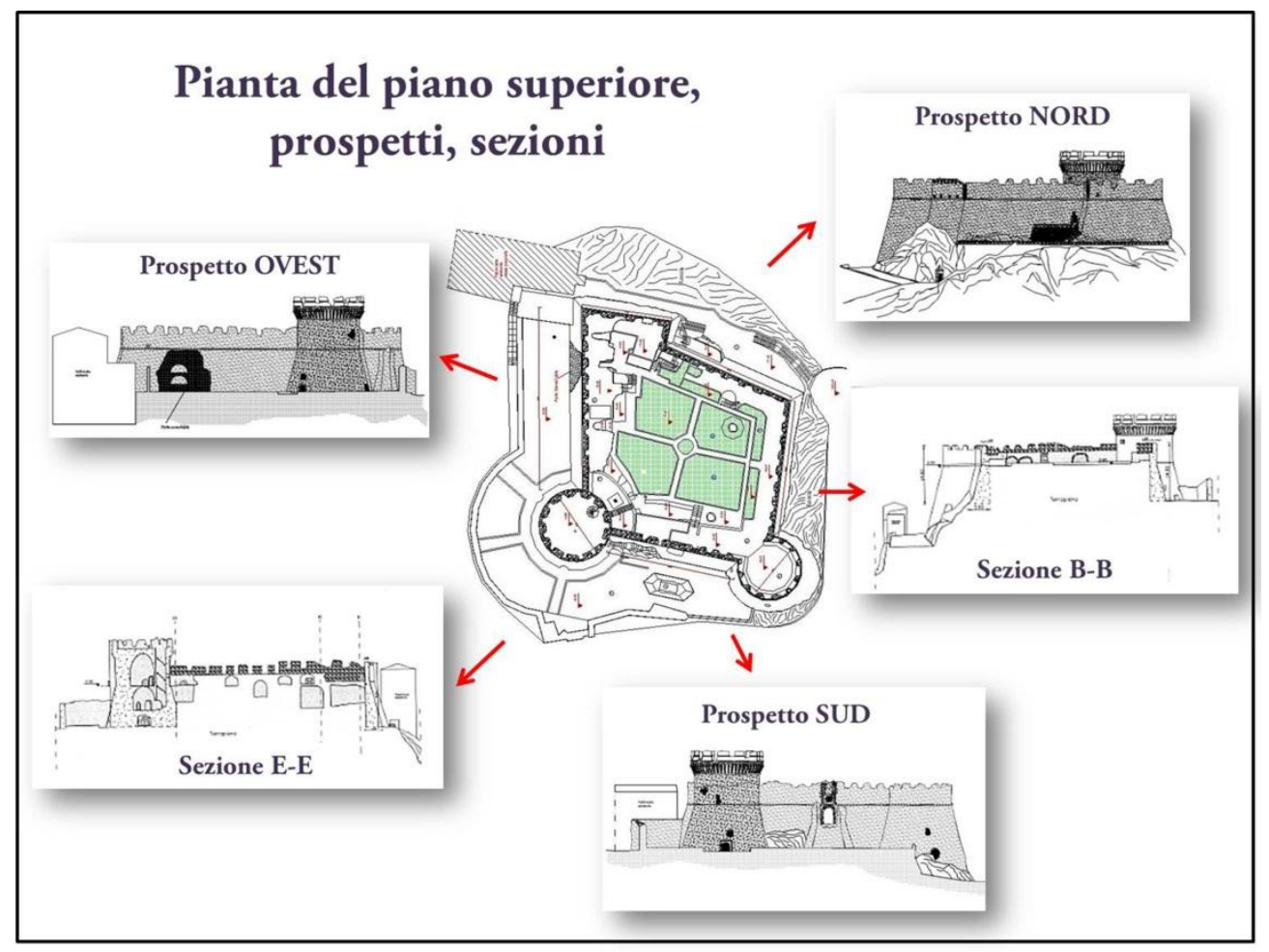

Fig. 7 - Il Castello di Belvedere: Pianta, prospetti, sezioni 
Un percorso stretto conduceva a tre celle e una scala a chiocciola portava alla sommità del castello, adesso entrambi inaccessibili. La parte superiore centrale ospitava dei giardini mentre sul perimetro erano collocati i sentieri di guardia. Dal giardino attraverso due porte si potevano raggiungere dei vani terrieri e dei sotterranei dove un nascondiglio portava alla cosiddetta torre di Paolo-Emilio attualmente crollata (Olivito, 2014).

\subsection{Campionamento ed esame macroscopico}

Nella redazione di un corretto progetto di conservazione e restauro risulta di fondamentale importanza, durante il processo di conoscenza del manufatto in questione, focalizzare l'attenzione sui materiali costitutivi eseguendone la loro caratterizzazione attraverso opportune tecniche di indagine diagnostica, al fine di indicare le tipologie di intervento più adeguate. Nel caso specifico, data la rilevanza storica e culturale del castello in esame, è stato necessario predisporre un piano di campionamento idoneo finalizzato a preservare interamente la struttura senza arrecarle alcun danno (Matteini ,2003).

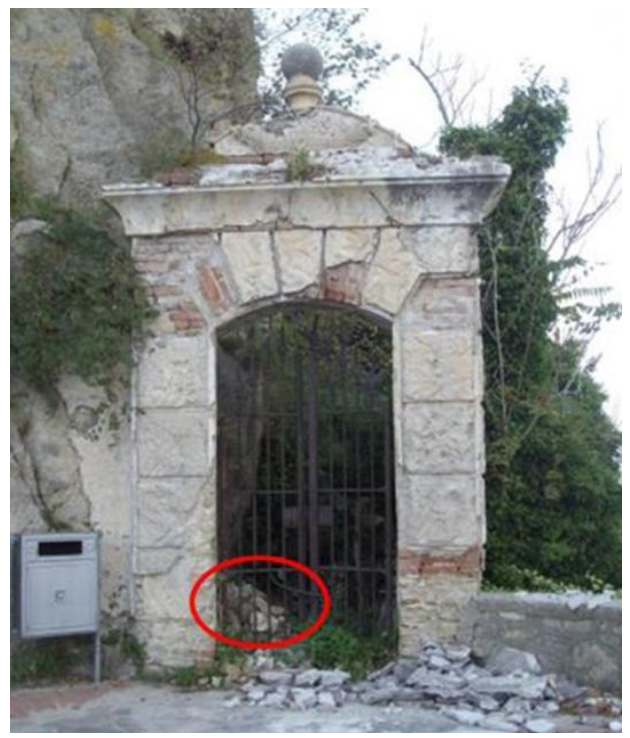

Fig. 8 - Punto di prelievo

Pertanto il campionamento è stato effettuato in modo del tutto non invasivo, prelevando nei pressi della struttura alcuni frammenti di materiale già distaccatosi dalla muratura per effetto di cedimenti avvenuti in precedenza.

I campioni, opportunamente fotografati e documentati, sono stati prelevati sul lato est del castello (Fig. 8).

Al fine di indirizzare al meglio le successive indagini di laboratorio, i campioni sono stati sottoposti preliminarmente ad un attento esame visivo che ha permesso di ottenere informazioni circa l'aspetto macroscopico degli stessi.

I campioni si presentano a prima vista composti da un materiale dall'aspetto granulare e sono caratterizzati da una superficie notevolmente alterata e frastagliata. In diverse zone sono visibili granuli di grosse dimensioni e di vario colore: rosa, verde, marrone, bianco, grigio e giallo.

\subsection{Analisi al SEM-EDS}

La caratterizzazione dei materiali campionati è stata effettuata utilizzando il microscopio elettronico a scansione unito al sistema di microanalisi EDS (SEM-EDS), una metodologia analitica che consente di ottenere informazioni non solo di carattere morfologico, necessarie queste per valutare l'aspetto superficiale e lo stato di degrado e di conservazione del materiale, ma anche di eseguire un'analisi chimica puntuale o areale indispensabile per definire la composizione e la natura del materiale (Gallone, 1989). Poiché le analisi condotte sui materiali campionati hanno portato all'identificazione della medesima composizione chimica, si è scelto di riportare di seguito i risultati delle analisi di un campione rappresentativo ovvero il campione C6 (Fig. 9).

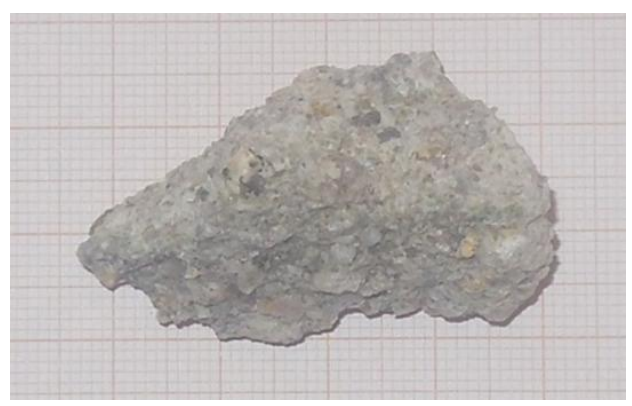

Fig. 9 - Campione C6 


\subsection{Analisi morfologica del campione C6}

Le immagini acquisite hanno messo in evidenza l'elevato stato di degrado in cui riversa il campione esaminato che appare fortemente disgregato e fratturato. La matrice si presenta composta da un insieme di microcristalli formatisi con molta probabilità in seguito a fenomeni di ricristallizzazione dovuti generalmente a processi di dissoluzione. Ulteriori ingrandimenti hanno permesso di identificare i granuli presenti, tra cui quarzo, feldspati e miche che appaiono notevolmente alterati (Fig. 10). Inoltre in molti punti della superficie, è stata rilevata la presenza di una patina di evidente natura biologica (Fig. 11a) che osservata con un ingrandimento maggiore (3000X) si presenta costituita da un insieme di colonie dalla forma sferica (Fig. 11b, Fig. 11c).

\subsection{Analisi chimica del campione $\mathrm{C} 6$}

L'analisi chimica è stata effettuata su una porzione di matrice mediante l'utilizzo del microscopio elettronico a scansione unito alla microsonda EDS che ha permesso di ricavarne lo spettro di fluorescenza.
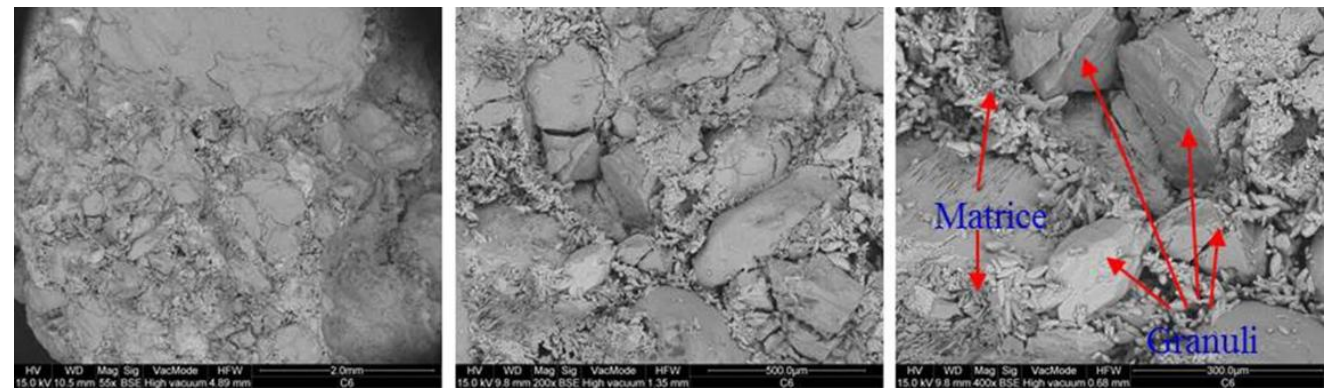

Fig. 10 -Visione d'insieme del campione (a); particolare dei granuli immersi nella matrice ricristallizzata (b, c)
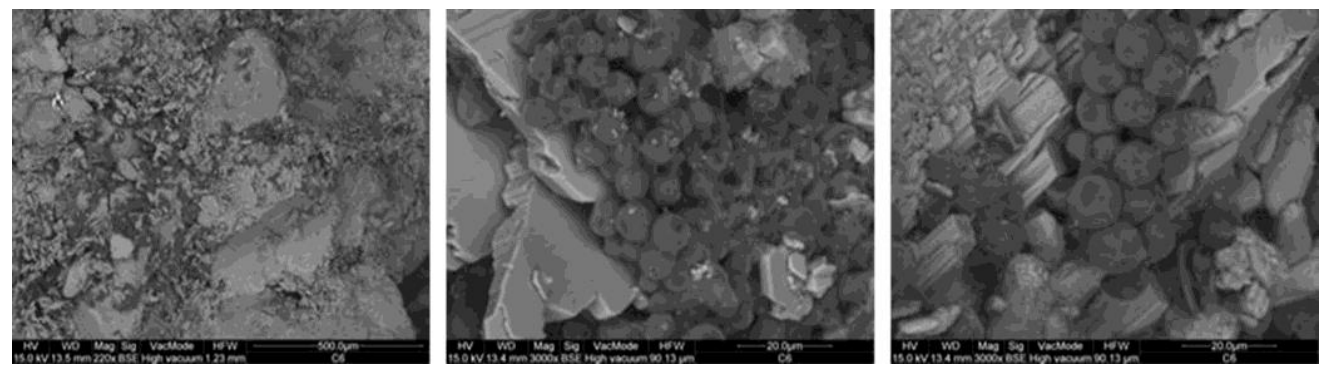

Fig. 11 - Presenza di patina biologica (a); Particolare delle colonie di microorganismi vegetali, ingrandimento $3000 \mathrm{X}(\mathrm{b}, \mathrm{c})$ 


\section{Conclusioni}

Con il presente lavoro si è voluto proporre, una metodica di studio innovativa finalizzata alla conservazione e valorizzazione dei manufatti di interesse storico-architettonico, che prevede l'elaborazione di un vero e proprio piano diagnostico inteso come l'insieme di tutta una serie di analisi ed azioni volte alla conoscenza del bene culturale sotto ogni profilo, da quello riguardante il contesto geografico a quello storico-architettonico fino a giungere allo studio dei materiali e del degrado. Tale metodica è stata illustrata e descritta attraverso la sua applicazione diretta su alcuni edifici di alto valore storico-architettonico nonché culturale, ovvero su alcuni dei più importanti castelli che si affacciano sul versante Tirrenico del territorio calabrese, concentrando l'attenzione su uno in particolare, il Castello di Belvedere Marittimo.

Nello specifico le analisi condotte sui materiali costitutivi del Castello di Belvedere Marittimo hanno permesso di evidenziare l'elevato stato di degrado in cui riversano le strutture murarie che necessiterebbero di mirati interventi di consolidamento al fine di impedire ulteriori crolli. Lo studio ha quindi permesso non solo di identificare elementi identitari e accomunanti nei vari casi analizzati ma anche di analizzare ciascun edificio in maniera completa fornendo tutte quelle informazioni necessarie ed indispensabili per poter intraprendere future azioni finalizzate alla conservazione e alla valorizzazione.

\section{References}

Aiello S., (2010), Il Castello di Pietramala - le Ragioni di un Restauro Strutturale, Calabria Letteraria Editrice.

Feiffer C., (1989), Il progetto di conservazione, Franco Angeli, Milano.

Gallone A., (1989), Analisi fisiche e conservazione, Franco Angeli, Milano.

Gattuso C., (2001) Conoscere per restaurare, Publiepa Edizioni, Cosenza.

Gattuso C. (2011), "Per un approccio razionale al piano diagnostico". IIth Convegno Internazionale AIES- Diagnosi per la conservazione e valorizzazione del Patrimonio Culturale, Ethos ed., Napoli.

Gattuso P., Gattuso C., Crisci G.M. (2010), "La ricerca storico-architettonica nel contesto del piano diagnostico", in Archeomatica, n.1- A\&C2000 editore, Roma.

Grisolia G. (1980), Belvedere Marittimo, Decollatura (CZ), 1980.

Matteini M., Moles A., (2003), Scienza e restauro, Nardini Editore, Firenze.

Olivito R. S., Gattuso C., Bencardino E., Codispoti R. (2014), Methodological approach for the restoration of the caste of Belvedere Marittimo (CS) Calabria, Atti del convegno "Best practices in heritage conservation and management. From the world to Pompeii. Le vie dei mercanti XII Forum Internazionale di Studi", Aversa | Capri.

Placanica A. (1999), Storia della Calabria Medievale, Gangemi, Roma.

Rogato E. (1992), "Belvedere Marittimo: Viaggio nel Passato", Editur Calabria- Belvedere Marittimo Associazione Socio-Culturale Ambiente e tradizione.

\section{Sitografia}

http://www.icastelli.it/castle-1235834741-castello_o_motta_di_santaniceto-it.php

www.viverefiumefrreddo.it

www.comunedifiumefreddobruzio.it 